\title{
Funcionamiento de la memoria de trabajo en usuarios de centros de la tercera edad
}

Amador, Lara y Ortiz 
Funcionamiento de la memoria de trabajo en usuarios de centros de la tercera edad

Working memory function in users of senior centers

(C) (1)
Dustin Ezequiel Amador Jiménez Universidad Nacional Autónoma de Nicaragua (UNANManagua).

https://orcid.org/0000-0001-6894-4997 dustin_amador@live.com

\section{Maria Elena Lara Sánchez \\ Licenciada en Psicología, Psicóloga independiente https://orcid.org/0000-0001-6271-1957 elenalarasanchez@gmail.com}

Ligia Walkiria Ortiz Gonzales Licenciada en Psicología. Psicóloga independiente. https://orcid.org/0000-0002-0949-2369 ligia.ortiz.gonzales@gmail.com

Recibido: 02 febrero 2020

Aceptado: 20 marzo 2020 Master en Psicología Clínica y de la Salud, Docente

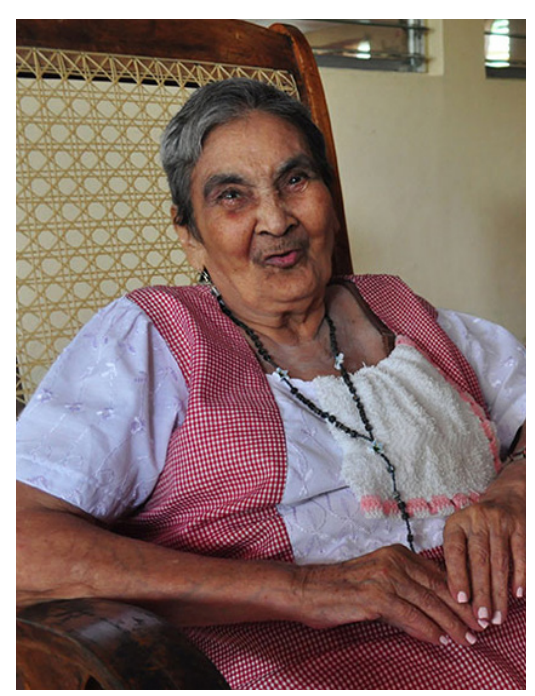

\section{RESUMEN}

Propósito: El estudio tiene como objetivo evaluar el nivel de funcionamiento de la memoria de trabajo en un grupo de usuarios de centros de la tercera edad del departamento de Carazo durante el segundo semestre del año 2019. Método: En el estudio se utilizó un enfoque cuantitativo, el cual permitió describir y establecer la relación de las variables en estudio; se utilizó un diseño no experimental, de corte transversal, con un alcance descriptivo-correlacional. La muestra está compuesta por 32 sujetos adultos mayores de 60 años, de ambos sexos, usuarios de Asilo Agustín Sánchez y el Comedor de la tercera edad La Purísima del departamento de Carazo. Se aplicó el Índice de Memoria de Trabajo (Escala de inteligencia para adultos de Weschsler, WAIS-III), compuesto por las subpruebas de Aritmética, Retención de dígitos y Ordenamiento de letras y números. Se hizo uso de estadística descriptiva y estadística inferencial, la cual permitió realizar el contraste de hipótesis. Resultados: Los resultados indican que existe deterioro de la memoria de trabajo en los participantes y presentan dificultades en la capacidad de manipular la información, tener control atencional, capacidad de planificación, organización, almacenamiento temporal y activo de la información verbal, visual y espacial.

\section{ABSTRACT}

Purpose: The study aims to assess the level of working memory function in a group of senior centers users from Carazo department during the second half of 2019. Method: The study used a quantitative approach, which allowed the description and establishment of the relationship of the variables under study; a non-experimental, cross sectional design was used, with a descriptive-correlational scope. The sample consists of 32 adults over the age of 60 , both sexes and users of Asylum Agustín Sánchez and La Purísima senior dining room. The Working Memory Index from the Weschsler Adult Intelligence Scale (WAIS III) was applied, composed by the subtests Arithmetic, Digit retention and Letters and numbers sorting. Descriptive and inferential statistics were used, which allowed the hypothesis contrast to be realized. Results:

\section{PALABRAS CLAVE}

\author{
Memoria de trabajo. Tercera edad.
}

\section{KEYWORDS}

Working memory, seniors. 
Results indicate that there is working memory difficulties in participants and presents problems in the ability to manipulate information, have attentional control, planning capacity, organization and temporary and active storage of verbal, visual and spatial information.

\section{Introducción}

El presente estudio tiene como objetivo principal evaluar el nivel de funcionamiento de la memoria de trabajo en un grupo de usuarios de centros de la tercera edad durante el segundo semestre del año 2019, para ello se toma en cuenta la edad y el sexo de los participantes. El estudio fue realizado en el Asilo Agustín Sánchez de la ciudad de Jinotepe y el Comedor de la tercera edad La Purísima de la ciudad de Diriamba, en el departamento de Carazo. Estos centros se encargan de brindar atención social a personas mayores de 60 años.

La investigación tiene como objeto de estudio el funcionamiento de la memoria de trabajo en sujetos de la tercera edad. La memoria es una función cognitiva que permite registrar, codificar, almacenar y recuperar información previamente almacenada (Portellano, 2005). De forma específica, la memoria de trabajo es un sistema de almacenamiento en tiempo real con capacidad limitada, que permite manipular las información facilitando el cumplimiento de varias tareas cognoscitivas de modo simultáneo, como el aprendizaje, el razonamiento, la comprensión y la resolución de problemas, gracias al mantenimiento y disponibilidad temporal de los datos (Baddeley, 1990, en Ostrosky y Flores, 2008) (Ramos, Sopena y Gilboy, 2007).

En la vejez se producen cambios físicos, psicológicos y sociales que pueden condicionar la calidad de vida de las personas. El envejecimiento normal se asocia con un menor rendimiento en la capacidad de memoria operativa y aprendizaje, una reducción en la velocidad de procesamiento de la información, razonamiento lógico y deductivo, así como de la capacidad de resolución de problemas (González y Muñoz, 2008) (Ardila y Rosselli, 2007), aunque estos cambios no representan un deterioro clínicamente significativo en la calidad de vida. Sin embargo, un el deterioro acelerado del funcionamiento en la memoria de trabajo se ha asociado a deterioro cognitivo de etiologías diversas como abandono, falta de estimulación cognitiva, trastornos neurodegenerativos, deficiencias nutricionales, alteraciones metabólicas o accidentes cerebrovasculares, entre otros (Ibíd.). Por lo tanto, este estudio tiene como hipótesis que existe un deterioro en el funcionamiento de la memoria de trabajo, y este se relaciona con la edad y el sexo de los participantes, por lo que es necesario estudiar detenidamente esta función cognitiva ya que con esta información las instituciones encargadas de abordar este tipo de problemáticas podrán diseñar planes terapéuticos para disminuir el deterioro cognitivo basados en evidencia científica.

\section{Método}

El estudio es una investigación aplicada, se utilizó un enfoque cuantitativo, el cual permitió describir y establecer la relación de las variables en estudio; se utilizó un diseño no experimental, de corte transversal, con un alcance descriptivo-correlacional. Participantes: La muestra está compuesta por 32 sujetos adultos de ambos sexos (16 varones y 16 mujeres) usuarios de Asilo Agustín Sánchez y el Comedor de la tercera edad La Purísima. El muestreo es de tipo no probabilístico o dirigido, ya que la elección de los participantes no depende de la probabilidad sino de las características de la investigación. Instrumentos: Se aplicó el Índice de Memoria de Trabajo (Escala de inteligencia para adultos de Weschsler, WAIS-III), compuesto por las subpruebas de Aritmética, Retención de dígitos 
y Ordenamiento de letras y números. Procedimiento: Para el análisis de los datos obtenidos se empleó procedimientos estadísticos descriptivos como Medidas de tendencia central y Distribución de frecuencia; para realizar la prueba de hipótesis y establecer el nivel de relación estadística entre las variables se empleó estadística inferencial, con las pruebas no paramétricas Chi cuadrada (X2) y la Prueba exacta de Fisher, en las que se asumió un Nivel de confianza del 95\% $(\alpha \leq 0.05)$.

\section{Resultados}

Memoria de trabajo: Según Ardila y Rosselli (2007), la memoria de trabajo se ve afectada con el envejecimiento, al reducirse esta función se limita la capacidad para almacenar, monitorear y manipular información mientras se realiza una tarea cognitiva en tiempo real, se necesita más tiempo y más ensayos para aprender y resolver problemas de la vida cotidiana. Cuando el deterioro de esta función es clínicamente significativo, puede ser un indicador de demencia y provocar discapacidad. En la Tabla 1 se pueden observar los resultados con respecto a esta variable.

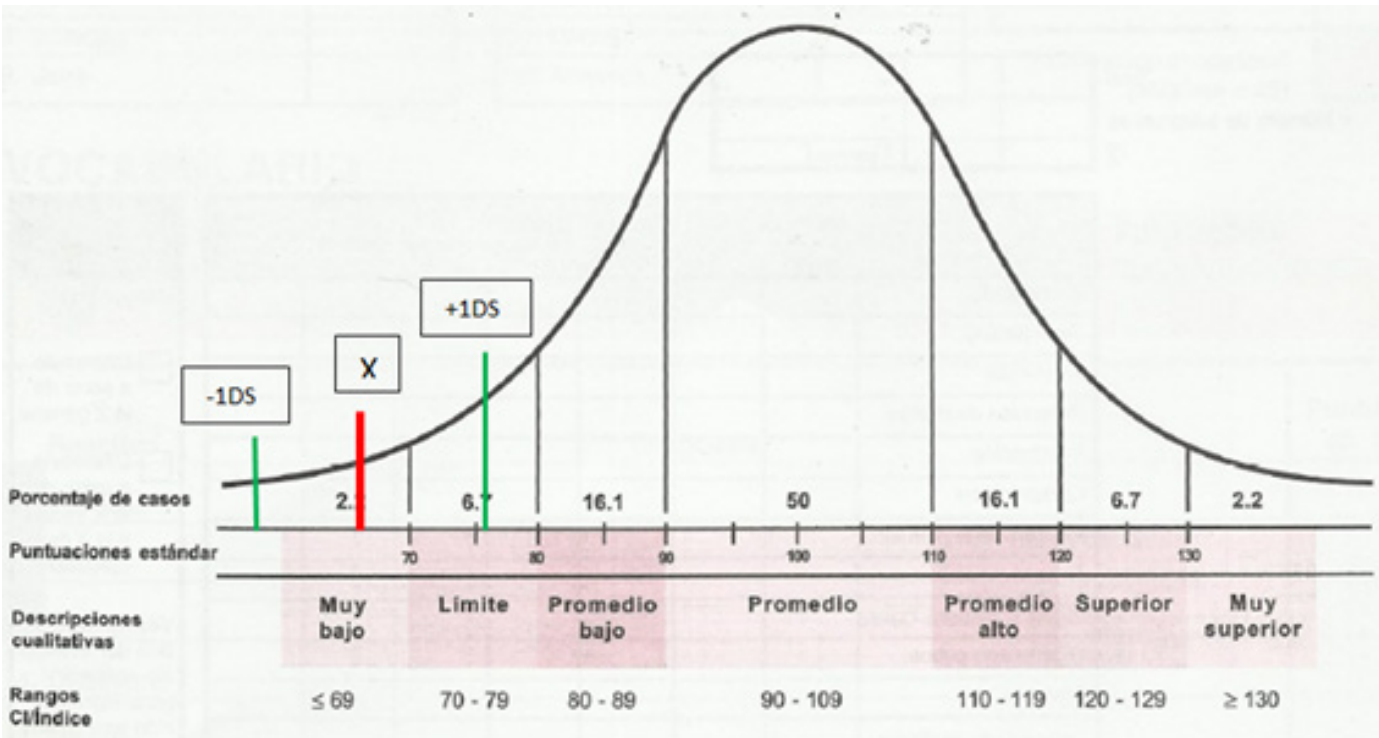

Al aplicar las subpruebas para obtener el Índice de Memoria de Trabajo, los participantes obtuvieron una media aritmética $(X)$ de 64,78 y una desviación estándar (DS) de 11,3. Estas puntuaciones indican que la mayoría de los sujetos obtuvieron puntuaciones estándar entre 53.48 (-1DS) y 76.08 (+1DS) unidades. Como se puede observar en la Figura 1, la media aritmética de la distribución normal de la escala del WAIS III es de 100 puntos con una desviación estándar de 10 unidades, en la de los sujetos participantes se ubica 3 desviaciones estándar por debajo del promedio de puntuaciones obtenidas por la mayoría de sujetos en los mismos rangos de edad. 


\section{Estadísticos descriptivos}

\begin{tabular}{|l|c|c|c|c|c|}
\hline & $N$ & Mínimo & Máximo & Media & $\begin{array}{l}\text { Desviación } \\
\text { estáandar }\end{array}$ \\
\hline $\begin{array}{l}\text { Índice de Memoria de } \\
\text { Trabajo } \\
\text { N válido ( po lista) }\end{array}$ & 32 & 50 & 85 & 64.78 & 11,373 \\
\hline
\end{tabular}

Por otra parte, los niveles de funcionamiento de la memoria de trabajo pueden apreciarse en la figura 2.

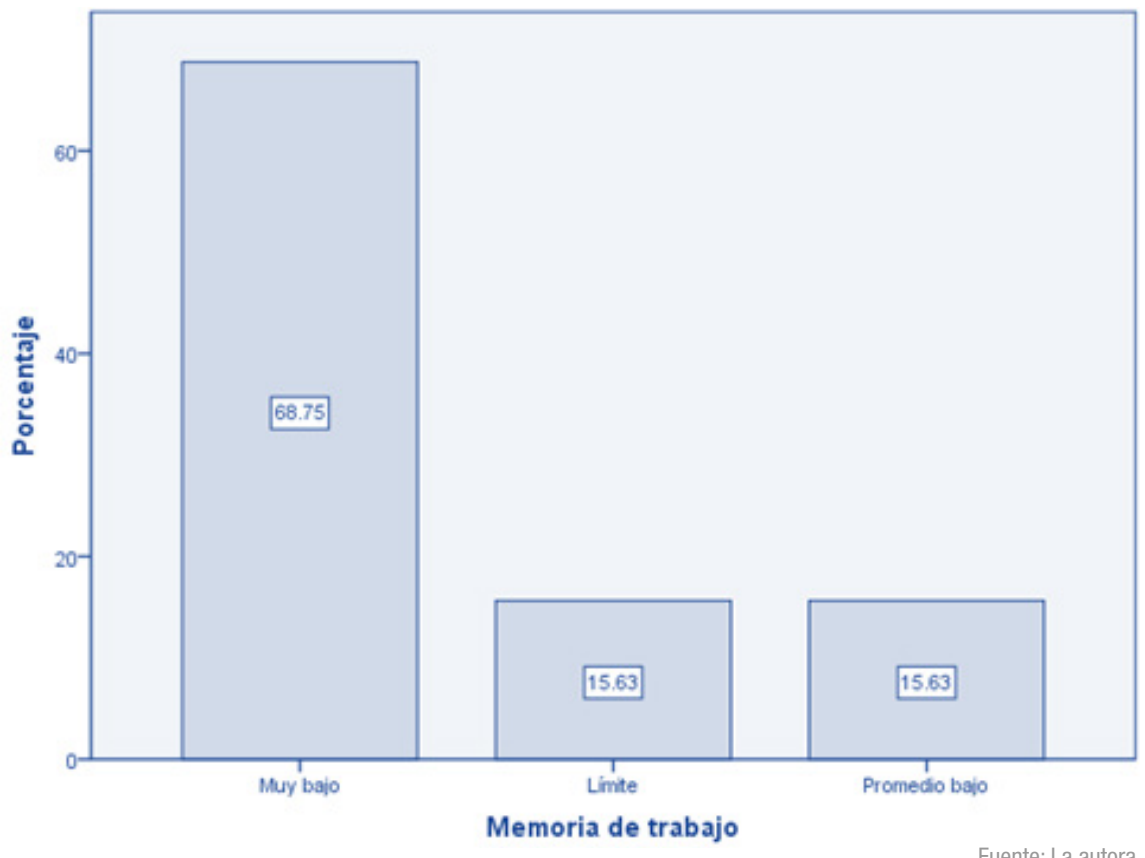

Se puede observar que el $68.75 \%$ de los sujetos de estudio tienen un nivel de funcionamiento muy bajo de esta capacidad cognitiva, el $15.63 \%$ de la muestra se ubica en la categoría límite y el $15.63 \%$ en el promedio bajo. Los resultados indican que existe deterioro de la memoria de trabajo en la mayoría de los participantes y presentan problemas en el cálculo, concentración, aprendizaje y razonamiento lógico.

Memoria de trabajo y edad: La edad avanzada se ha asociado de forma consistente con el deterioro del funcionamiento de la memoria de trabajo. En la figura 3 se puede apreciar que $75 \%$ de los participantes entre las edades de 60-69 años tienen un funcionamiento muy bajo de la memoria de trabajo, el $12.50 \%$ tiene un funcionamiento promedio bajo y el $12.50 \%$ se encuentra en la categoría límite. Por otro lado, entre los participantes con edades de $70-79$ años, el 67\% tiene un funcionamiento muy bajo de la memoria de trabajo, el $33 \%$ restante presenta un nivel de funcionamiento promedio bajo. 
Por último, el $57 \%$ de los sujetos entre $80-89$ años tienen un funcionamiento muy bajo en esta capacidad cognitiva, mientras que el $43 \%$ de este grupo de edad tiene un funcionamiento límite.

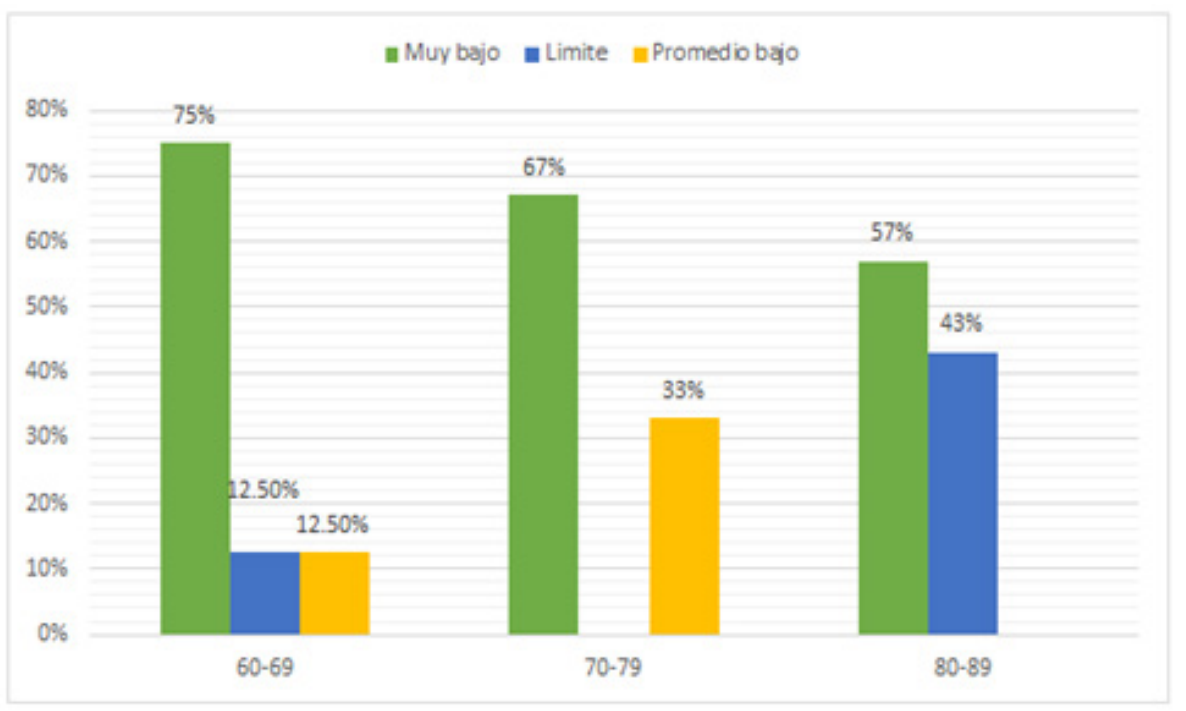

Fuente: La autora

En la Tabla 2 se puede observar el nivel de relación estadística que existe entre la memoria de trabajo y la edad de los participantes.

\section{Memoria de trabajo y edad}

\begin{tabular}{|l|c|c|c|c|}
\hline $\begin{array}{l}\text { Indice de Memoria de } \\
\text { Trabajo }\end{array}$ & Valor & gl & $\begin{array}{c}\text { Sig. asinfótica } \\
\text { (bilateral) }\end{array}$ & $\begin{array}{c}\text { Sig. exacta } \\
\text { (bilateral) }\end{array}$ \\
\hline Chi-cuadrado de Pearson & $8.062^{\mathrm{a}}$ & 4 & .089 & .094 \\
Razón de verosimilitudes & 9.055 & 4 & .060 & .091 \\
Estadisticos exactos de Fisher & 6.378 & & & .124 \\
N de casos válidos & 32 & & & \\
\hline
\end{tabular}

En la prueba de Chi cuadrada de Pearson se obtuvo $\mathrm{X} 2=8.062$, $\mathrm{gl}=4$ y $\alpha=0.089$, mientras que en la prueba exacta de Fisher refleja $F=6.378, \alpha=0.124$, en ambos casos el nivel de significancia es mayor a 0.05 , por tanto no existe relación estadísticamente significativa entre memoria de trabajo y edad de los participantes.

Memoria de trabajo y sexo: En la Figura 4 se representa el nivel de funcionamiento de la memoria de trabajo en función del sexo, el $56 \%$ de los sujetos masculinos tienen un nivel de funcionamiento muy bajo, el 19\% tienen un nivel de funcionamiento límite y un $25 \%$ dentro de la categoría promedio bajo. Por otro lado, el $81 \%$ del sexo femenino tiene un nivel de funcionamiento muy bajo, el $13 \%$ un nivel límite y otro $6 \%$ un nivel promedio bajo. 


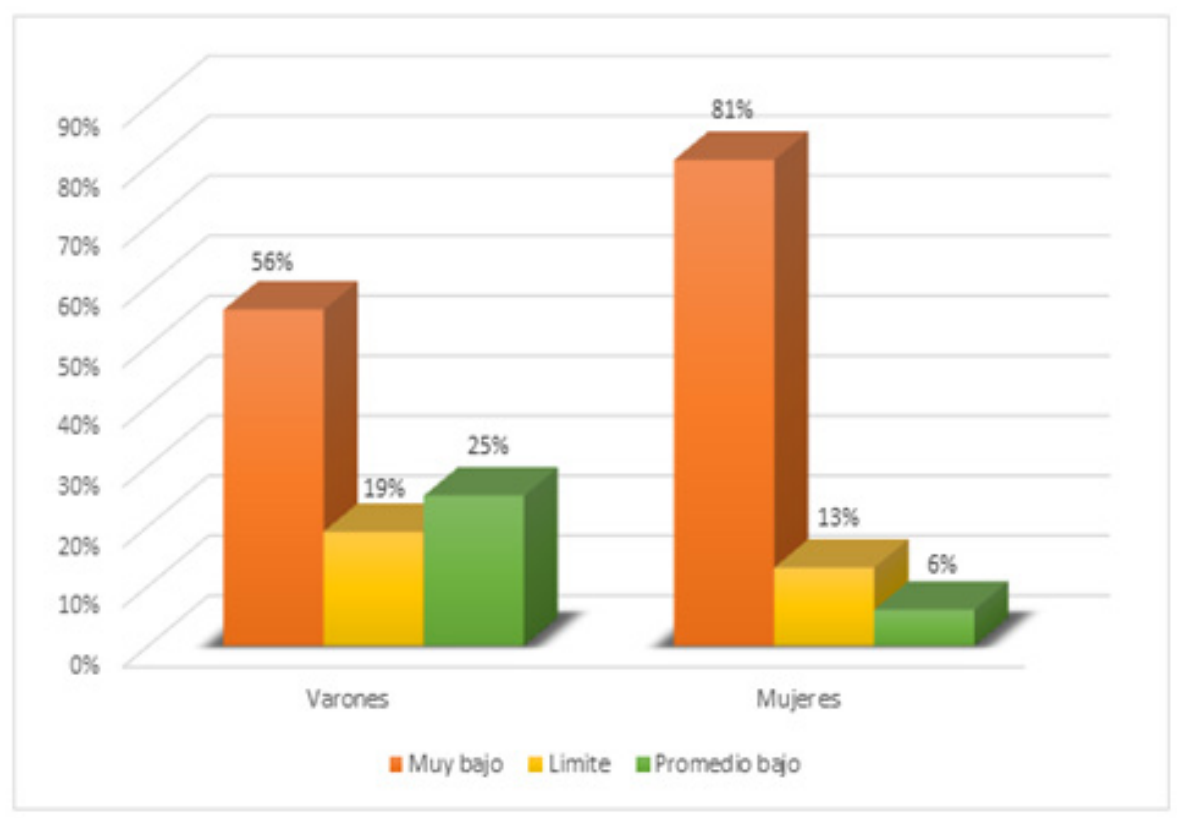

Fuente: La autora

El nivel de funcionamiento tanto para el sexo femenino como para el masculino se encuentra dentro de la categoría Muy bajo lo que indica que ambos sexos presentan problemas en la memoria de trabajo la cual hace uso de la memoria inmediata, atención, percepción y concentración.

En la Tabla 3 se puede observar el nivel de relación estadística que existe entre la memoria de trabajo y el sexo de los participantes.

\section{Memoria de trabajo y sexo}

\begin{tabular}{|l|c|c|c|c|}
\hline & Valor & gl & $\begin{array}{c}\text { Sig. asinfótica } \\
\text { (bilateral) }\end{array}$ & $\begin{array}{c}\text { Sig. exacta } \\
\text { (bilateral) }\end{array}$ \\
\hline Chi-cuadradi de Pearson & $2.727^{\mathrm{a}}$ & 2 & .256 & .335 \\
Razón de verosimilitudes & 2.860 & 2 & .239 & .335 \\
Estadístico exacto de Fisher & 2.623 & & & .335 \\
N de casos válidos & 32 & & & \\
\hline
\end{tabular}

En cuanto a la prueba de Chi cuadrada, se obtuvo $\mathrm{X} 2=2.727, \mathrm{gl}=2 \mathrm{y} \mathrm{\nabla}=0.335$, mientras que la prueba exacta de Fisher refleja $F=2.623$ y $\otimes=0.335$. Nuevamente, el nivel de significancia es mayor a 0.05 , por tanto no existe relación estadísticamente significativa entre memoria de trabajo y el sexo de los participantes, ambos sexos tienen dificultades significativas con respecto a esta capacidad cognitiva. 


\section{Discusión}

Los resultados del estudio indican que existe deterioro de la memoria de trabajo en los participantes del estudio, lo que significa que tienen muy poca capacidad de manipular la información, tener control atencional, capacidad de planificación, organización, almacenamiento temporal y activo de la información verbal, visual y espacial.

Durante el transcurso de la acción, el sujeto necesita disponer de una representación mental tanto del objetivo como de la información relevante, no sólo acerca del estado actual sino también con relación a la situación futura (Herreras, 2007), el deterioro de la memoria de trabajo dificulta el procesamiento de la información dando como resultado un peor rendimiento en tareas cognitivas y acciones no automatizadas. La memoria de trabajo es un sistema formado por tres elementos: el sistema ejecutivo central, el bucle fonológico y la agenda visoespacial (Tirapu, Muñoz, y Pelegrín, 2002).

El ejecutivo central el cual coordina las actividades de dos sistemas subordinados, se ocupa de los aspectos atencionales y estratégicos, y su misión sería controlar, coordinar y supervisar las actividades realizadas por el sistema cognitivo. Se considera un elemento nuclear porque gobierna los sistemas de memoria (Ostrosky y Lozano, 2011). Otro elemento es el bucle fonológico, el cual se encarga de mantener en línea información de tipo verbal a través de la repetición articulatoria; este sistema procesa y retiene la información oral durante uno o dos segundos, y de un mecanismo de repetición subvocálico, que fortalece la huella de la información contenida en el almacén. Así, se alarga el tiempo de permanencia de la información en la memoria de trabajo y se evita su decaimiento; además, el proceso de repaso articulatorio participa en la transformación de los códigos no fonológicos en fonológicos, lo que es necesario para su registro en el bucle (ibíd.). La memoria de trabajo también incluye una agenda visoespacial, este componente se encarga de elaborar y manipular información visual y espacial, implicada en la aptitud espacial y en tareas que suponen memoria espacial (ibíd.).

El déficit en la capacidad de la memoria de trabajo se disemina en los tres elementos que componen el sistema (Tirapu, Muñoz, y Pelegrín, 2002). En el ejecutivo central se presenta dificultades para administrar la atención y las estrategias de control, coordinación y supervisión de las actividades realizadas por el sistema cognitivo de memoria. Por ejemplo, se manifiestan dificultades para administrar los recursos atencionales cuando se realizan dos o más tareas simultáneas y acceder a información almacenada en la memoria a largo plazo, seleccionar las estrategias adecuadas de evocación y recuerdo. En cuanto al bucle fonológico, las deficiencias se manifiestan en dificultades de procesamiento y retención de la información oral durante unos segundos. Se acorta el tiempo de permanencia de la información en la memoria de trabajo y se produce su decaimiento. Finalmente, en la agenda visoespacial las deficiencias se presentan en la poca capacidad de elaborar y manipular información visual y espacial, implicadas en la realización de tareas perceptivo motoras. 
En términos generales, la interferencia en la memoria de trabajo se traduce en peores prestaciones en las tareas de razonamiento e ineptitud en resolución de problemas complejos, por tanto se presentan dificultades al momento de realizar aprendizajes de nuevos comportamientos.

Una de las principales explicaciones de las alteraciones de memoria se relaciona con dificultades de control inhibitorio atencionales sobre los procesos de memoria de trabajo (Hasher et al., 1999). El envejecimiento se caracterizaría por la presencia de información irrelevante y falta de autocontrol de los procesos de memoria de trabajo. La falta de control inhibitorio cognitivo congestionaría la capacidad de la memoria, disminuyendo su eficacia para almacenar y manipular nueva información de forma activa y en tiempo real, además de incrementar la interferencia en el proceso de evocación de información.

Finalmente, es necesario destacar que en términos neurobiológicos, el lóbulo frontal y específicamente, la corteza prefrontal dorsolateral es la región cerebral más involucrada en la memoria de trabajo. Las lesiones prefrontales, especialmente las de la región dorsolateral afectan el funcionamiento normal de la memoria de trabajo lo que compromete la integridad de los procesos cognitivos (Herreras, 2007), por lo tanto, los resultados del estudio son indicadores de deterioro cognitivo y la posible presencia de alteraciones neuroanatómicas y/o neurofisiológicas en la corteza prefrontal de los participantes con alteraciones graves en la el funcionamiento de la memoria de trabajo.

En cuanto al nivel del funcionamiento de la memoria de trabajo y su relación con la edad, el bajo nivel predomina en todos los grupos. Aunque estadísticamente no se encontró relación significativa, a nivel descriptivo se puede observar que los sujetos entre 60 y 69 años tienen un mayor deterioro de la memoria de trabajo en comparación con los participantes entre 80 y 80 . Este resultado se contradice con los resultados de diversas investigaciones (Portellano y García, 2015) que indican la relación entre el deterioro de la memoria y las edades avanzadas, esto podría explicarse debido a diversos factores como la frecuencia de nivel educativo, actividad intelectual, interacción social, nutrición y presencia de enfermedades médicas. Dados estos resultados, es recomendable realizar la comparación con personas de mediana edad.

Con respecto al nivel del funcionamiento de la memoria de trabajo y el sexo, no existe relación estadísticamente significativa entre la variable, sin embargo, a nivel descriptivo se observa que las mujeres tienen un mayor nivel de deterioro de la memoria de trabajo, lo que coincide estudios que sugieren la existencia de diferencias en esta capacidad cognitiva, las cuales se atribuyen de forma preliminar a diferencias hormonales (Nastayashchada y López, 2015).

\section{Conclusiones}

Los resultados indican que existe deterioro de la memoria de trabajo en los participantes y presentan dificultades en la capacidad de manipular la información, tener control atencional, capacidad de planificación, organización, almacenamiento temporal y manipulación de la información verbal, visual y espacial.

En el abordaje de los niveles de funcionamiento de la memoria de trabajo, se encontró que la mayoría de los participantes de este estudio presentan un nivel muy bajo, lo que es un indicador de deterioro cognitivo significativo, el cual tiene repercusiones en la calidad de vida de las personas. 
Con respecto al funcionamiento de la memoria de trabajo, la edad y el sexo, no se encontró relación estadísticamente significativa entre las variables en cuestión, ambos sexos tienen dificultades significativas con respecto a esta capacidad cognitiva.

\section{Referencias bibliográficas}

- Ardila, A., y Rosselli, M. (2007). Neuropsicología Clínica. México: Editorial El Manual Moderno.

- González, B., y Muñoz, E. (2008). Estimulación de la memoria en personas mayores. Madrid: Editorial Síntesis, S.A.

- Hasher, L., Zacks, R., y Rahhal, T. (1999). Timing instructions, and inhibitory control: some missing factors in the age and memory debate. Gerontology, 45. 355-357.

- Herreras, E. (2007). Función ejecutiva: evaluación y rehebilitación neurológica. Revista de Educación, 21 (9), 291-300.

- Nastayashchada, E., y López, L. (2015). Diferencia entre hombres y mujeres en memoria de trabajo. Revista Neuropsicología, Neuropsiquiatría y Neurociencias, 15 (2), 35-51.

- Ostrosky, F., y Lozano, A. (2011). Desarrollo de las funciones ejecutivas y la corteza prefrontal. Revista Neuropsicología, Neuropsiquiatría y Neurociencias, 11 (1), 159-172.

- Portellano, J. (2005). Introducción a la Neuropsicología. Madrid: Editorial McGraw-Hill.

- Portellano, J., y García, J. (2015). Neuropsicología de la atención, las funciones ejecutivas y la memoria. Madrid: Editorial Síntesis, S.A.

- Ramos, P., Sopena, J., y Gilboy, E. (2007). Memoria de trabajo, atención y composicionalidad. Anuario de Psicología, 38 (1), 93-116.

- Tirapu, J., Muñoz, J., \& Pelegrín, C. (2002). Funciones ejecutivas: necesidad de una integración conceptual. Revista de Neurología, 34 (7), 673-685.

Dustin Ezequiel Amador Jiménez Master en Psicología Clínica y de la Salud, Docente Universidad Nacional Autónoma de Nicaragua (UNANManagua) https://orcid.org/0000-0001-6894-4997 dustin_amador@live.com

María Elena Lara Sánchez Licenciada en Psicología, Psicóloga independiente https://orcid.org/0000-0001-6271-1957 elenalarasanchez@gmail.com

Ligia Walkiria Ortiz Gonzales

Licenciada en Psicología. Psicóloga independiente. https://orcid.org/0000-0002-0949-2369 ligia.ortiz.gonzales@gmail.com 